www.nature.com/pj

\title{
Dielectric relaxation study of the crystalline chain motion of poly(vinylidene fluoride) under carbon dioxide
}

\author{
Yoshiko Yamamura, Kazuo Yoshida, Kiyu Kawate, Noboru Osaka and Hiromu Saito
}

Polymer Journal (2010) 42, 419-422; doi:10.1038/pj.2010.19; published online 24 March 2010

Keywords: carbon dioxide; crystalline chain motion; in situ dielectric relaxation; poly(vinylidene fluoride)

\section{INTRODUCTION}

Carbon dioxide $\left(\mathrm{CO}_{2}\right)$ can dissolve into a free volume of amorphous polymers because of the low viscosity and/or high diffusivity of $\mathrm{CO}_{2}{ }^{1}$ The dissolved $\mathrm{CO}_{2}$ causes a plasticization effect such as dilation, ${ }^{2}$ depression of glass transition temperature $\mathrm{e}^{3,4}$ and decrease in viscosity. ${ }^{5}$ Hence, for example, $\mathrm{CO}_{2}$ is used as a plasticizer in polymer processing. The plasticization effect on amorphous polymers has been verified in terms of molecular motion by using in situ dielectric relaxation measurement under $\mathrm{CO}_{2}$; that is, the dielectric relaxation spectrum for the amorphous chain $\left(\alpha_{\mathrm{a}}\right.$ relaxation) shifts to a higher frequency by pressurized $\mathrm{CO}_{2}$, suggesting that the molecular motion of the amorphous chain is accelerated by the plasticization effect of $\mathrm{CO}_{2} \cdot{ }^{6,7}$

In crystalline polymers, a crystalline region coexists with an amorphous one. The packing of the chain in the crystalline region (crystalline chain) is tight in crystalline polymers, whereas the chain is randomly arranged and free volume exists in amorphous polymers. Hence, the sorption and solubility of $\mathrm{CO}_{2}$ into crystalline polymers are much smaller than those of amorphous polymers, suggesting that $\mathrm{CO}_{2}$ is insoluble in the crystalline region. ${ }^{3,8}$ Because $\mathrm{CO}_{2}$ is insoluble in the crystalline region, whereas it dissolves in the amorphous region, dissolved $\mathrm{CO}_{2}$ in the molten state is excluded from the crystal growth front during crystallization. Owing to the exclusion, characteristic porous structures such as layered and rod-like ones are obtained when the bubbles nucleate and grow by supersaturation of the excluded $\mathrm{CO}_{2}$ in the constrained intercrystalline amorphous region. ${ }^{9}$ On the other hand, a decline in melting temperature is observed under $\mathrm{CO}_{2}$ in several crystalline polymers, suggesting the plasticization effect of $\mathrm{CO}_{2}$ on the crystalline chain. ${ }^{4,10,11}$ Thus, the solubility and plasticization effects of $\mathrm{CO}_{2}$ on the crystalline chain are controversial. However, thus far, the solubility of $\mathrm{CO}_{2}$ in the crystalline chain has not been clarified on a molecular scale.

If $\mathrm{CO}_{2}$ is dissolved in the crystalline region, the molecular motion of the crystalline chain should be accelerated by its plasticization effect.
Dielectric relaxation measurement is a powerful tool to estimate the molecular motion of the crystalline chain under $\mathrm{CO}_{2}$. In this paper, we investigate the molecular motion of the crystalline chain by in situ dielectric relaxation measurement of a crystalline polymer under $\mathrm{CO}_{2}$ at various pressures. Here, we chose poly(vinylidene fluoride) (PVDF) as the crystalline polymer because the decline in its melting temperature can be observed under $\mathrm{CO}_{2}{ }^{11}$ and the dielectric relaxation spectra of the crystalline chain $\left(\alpha_{c}\right.$ relaxation) are seen near $120^{\circ} \mathrm{C}$ under air at ambient pressure. ${ }^{12,13}$ The dissolution of $\mathrm{CO}_{2}$ in the crystalline region is discussed in terms of the molecular motion of the crystalline chain.

\section{EXPERIMENTAL PROCEDURE}

The PVDF powder used in this study was supplied by Kureha Chemical Industry Co., Ltd (Tokyo, Japan) (KF-1000, $\left.M_{\mathrm{w}}=250000\right)$. To obtain a crystallized film specimen with a thickness of $0.4 \mathrm{~mm}$, the PVDF powder was compression molded between two cover glasses at $200{ }^{\circ} \mathrm{C}$ for $5 \mathrm{~min}$, and then gradually cooled to room temperature.

In situ dielectric relaxation measurement under high-pressure $\mathrm{CO}_{2}$ was taken using a specially designed custom-made high-pressure cell constructed of stainless steel with an inner volume of $300 \mathrm{ml}$ equipped with two sapphire windows for through view, as schematically shown in Figure 1. The capacitors used for the dielectric relaxation measurement consisted of two indium tin oxide plates. The upper capacitor was hung from a shaft and the lower one was fixed at the lower part of the cell. As the magnet was attached at the upper end of the shaft in a hollow bolt and was held by a magnetic nut on the hollow bolt with screw thread, the upper capacitor could be moved up or down, thereby moving the shaft by rotating the magnetic nut on the hollow bolt. The film specimen was placed on the lower capacitor and high-pressure $\mathrm{CO}_{2}$ was injected into the cell at room temperature. After the specimen was kept in position for $2 \mathrm{~h}$ to dissolve $\mathrm{CO}_{2}$ in the specimen, the upper capacitor was moved downward until it adhered to the specimen as viewed through the sapphire window. Here, the capacitors were separated by a polyimide spacer of $0.4 \mathrm{~mm}$ to determine the thickness of the specimen. The temperature was then 


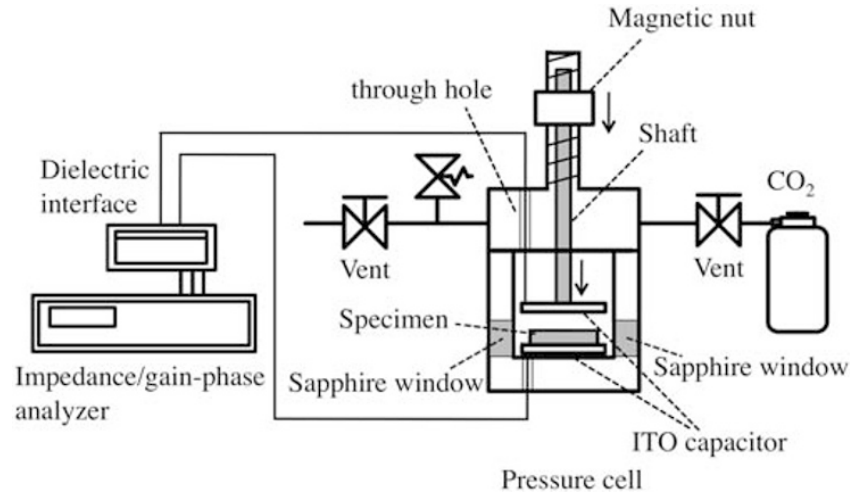

Figure 1 Schematic illustration of the instrumental setup for in situ dielectric relaxation measurement.

raised to $120^{\circ} \mathrm{C}$. The pressure of $\mathrm{CO}_{2}$ was kept constant during the heating process using a back pressure regulator. Dielectric measurements were performed with a Solartron 1260 Impedance/Gain-Phase Analyzer equipped with an impedance transformer (SI 1296 Dielectric Interface, Solartron, Farnborough, UK) under $\mathrm{CO}_{2}$ pressure up to $10 \mathrm{MPa}$ at a frequency, $f$, ranging from $10^{-1}$ to $10^{7} \mathrm{~Hz}$. The angular frequency, $\omega$, was obtained by $\omega=2 \pi f$.

\section{RESULTS AND DISCUSSION}

Figure 2 shows the angular frequency, $\omega$, dependence of the dielectric loss, $\varepsilon^{\prime \prime}$, of the crystallized PVDF at $120^{\circ} \mathrm{C}$ under various $\mathrm{CO}_{2}$ pressures, $P_{\mathrm{CO} 2}$. A peak is detected near $10^{5} \mathrm{~s}^{-1}$ and a broad shoulder is observed near $10^{2} \mathrm{~s}^{-1}$. The peak near $10^{5} \mathrm{~s}^{-1}$ is assigned to the crystalline chain relaxation $\left(\alpha_{c}\right.$ relaxation). ${ }^{12,13}$ As the $\alpha_{c}$ relaxation originates from rotating and twisting with a small lengthwise translation of the crystalline chain, ${ }^{14,15}$ this peak is expected to shift to a higher frequency when the crystalline chain motion is accelerated by dissolving $\mathrm{CO}_{2}$ in the crystalline region. On the other hand, the shoulder near $10^{2} \mathrm{~s}^{-1}$ is assigned to the relaxation of the interfacial polarization caused by trapping charge carriers at the crystal-amorphous interphase because of the permittivity difference between the amorphous and crystalline regions. ${ }^{16,17}$ A steep upturn with a straight line of slope -1 is seen at a low frequency below $10^{2} \mathrm{~s}^{-1}$. The steep upturn is assigned to ionic conductivity caused by the mobility of ionic impurities in the amorphous region. ${ }^{2,18}$

As shown in Figure 2b, the $\varepsilon^{\prime \prime}(\omega)$ curve in Figure 2a can easily be resolved into three parts. The peak for the $\alpha_{c}$ relaxation and the shoulder for the relaxation of the interfacial polarization are described by the Havriliak-Negami equation: ${ }^{16}$

$$
\varepsilon^{*}=\varepsilon_{\infty}+\sum_{j} \frac{(\Delta \varepsilon)_{j}}{\left[1+\left(i \omega \tau_{j}\right)^{\alpha_{j}}\right]^{\beta_{j}}}
$$

where $\varepsilon^{*}$ is the complex dielectric permittivity; $\varepsilon^{*}=\varepsilon^{\prime}-i \varepsilon^{\prime \prime}, \varepsilon_{\infty}$ is the limiting high-frequency permittivity; $\tau_{j}$ is the relaxation time of the $j$ th process; $(\Delta \varepsilon)_{j}$ is the dielectric strength of the $j$ th process; and $\alpha_{j}$ and $\beta_{j}$ are the parameters describing the broadening and asymmetry of the $j$ th relaxation time distribution. The upturn for the ionic conductivity is described by

$$
\varepsilon_{\text {ionic }}^{*}(\omega)=-\frac{i \sigma_{\text {ionic }}}{\omega \varepsilon_{0}}
$$

where $\sigma_{\text {ionic }}$ is the ionic conductivity and $\varepsilon_{0}$ is the permittivity of vacuum $\left(8.854 \mathrm{pFm}^{-1}\right)$.

Figure 3 shows the peaks for $\alpha_{c}$ relaxation and interfacial polarization at various $\mathrm{CO}_{2}$ pressures that are obtained from Figure 2 using
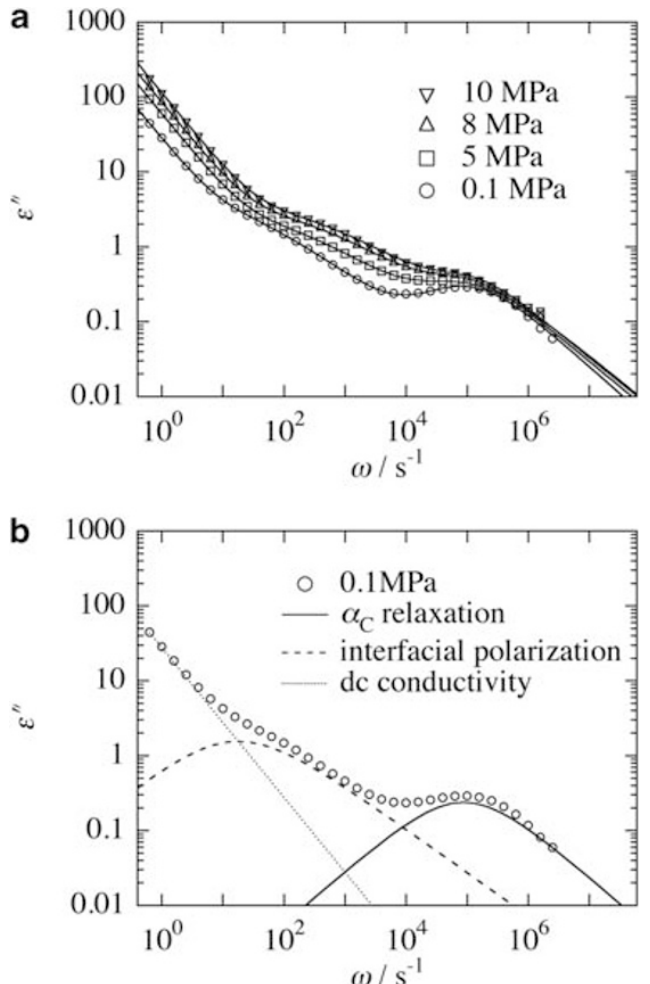

Figure 2 Frequency dependence of dielectric loss $\varepsilon^{\prime \prime}$. (a) Crystallized PVDF under various $\mathrm{CO}_{2}$ pressures at a temperature of $120^{\circ} \mathrm{C}$. The solid lines were obtained by the fitting procedure shown in (b). (b) Crystallized PVDF under air at ambient pressure $(0.1 \mathrm{MPa})$ at a temperature of $120^{\circ} \mathrm{C}$. The curve was fitted by Equation (1) for $\alpha_{\mathrm{c}}$ relaxation and interfacial polarization, and by Equation (2) for ionic conductivity.
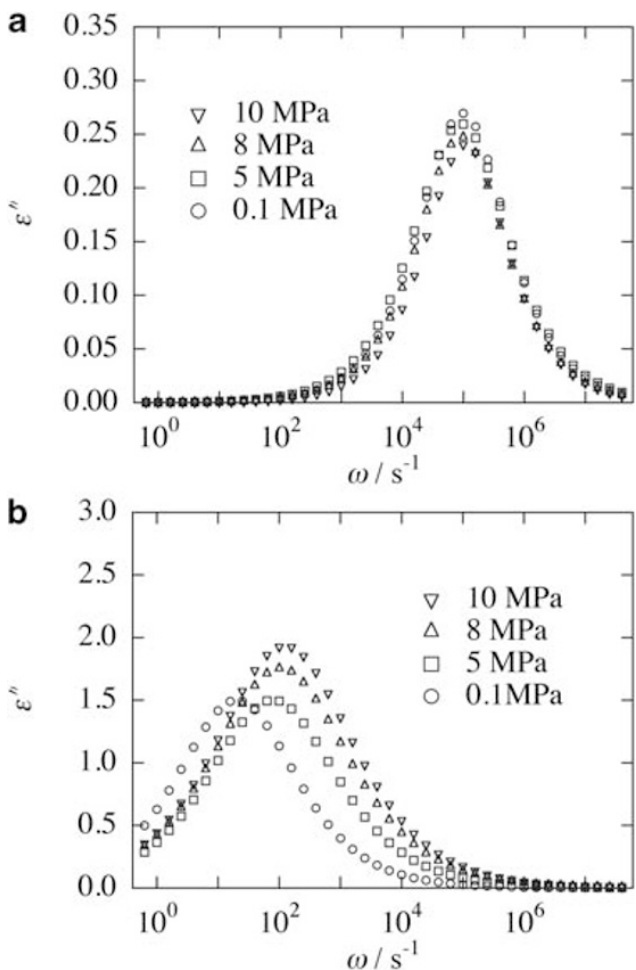

Figure 3 Frequency dependence of the dielectric loss of (a) $\alpha_{c}$ relaxation and (b) interfacial polarization in the crystallized PVDF under various $\mathrm{CO}_{2}$ pressures at a temperature of $120^{\circ} \mathrm{C}$. 


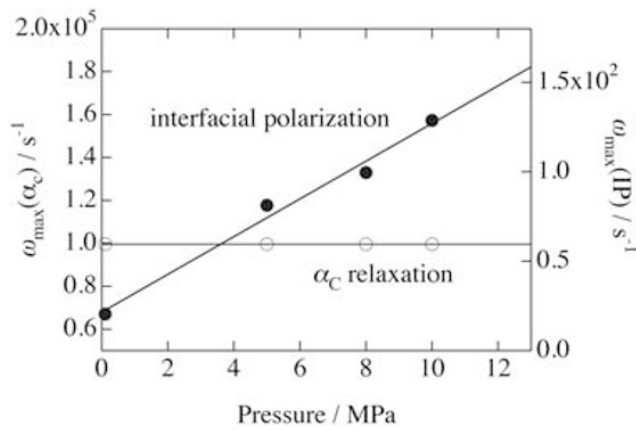

Figure 4 Pressure dependence of the frequency of the peak maximum of the dielectric loss of $\alpha_{c}$ relaxation and interfacial polarization in crystallized PVDF under $\mathrm{CO}_{2}$ at a temperature of $120^{\circ} \mathrm{C}$.

Equations (1) and (2). It was found that the shape and peak maximum for the $\alpha_{c}$ relaxation did not change, although pressurized $\mathrm{CO}_{2}$ was applied up to $10 \mathrm{MPa}$ (Figure $3 \mathrm{a}$ ). As shown in Figure 4 , the frequency of the peak maximum for the $\alpha_{\mathrm{c}}$ relaxation obtained from Figure $3 \mathrm{a}, \omega_{\max }\left(\alpha_{\mathrm{c}}\right)$, is almost constant near $10^{5} \mathrm{~s}^{-1}$ with $\mathrm{CO}_{2}$ pressure. The constant $\omega_{\max }\left(\alpha_{\mathrm{c}}\right)$ with $\mathrm{CO}_{2}$ pressure indicates that the molecular motion of the crystalline chain is not accelerated by applying pressurized $\mathrm{CO}_{2}$. This result is quite different from that observed in amorphous polymers in which accelerated chain motion by pressurized $\mathrm{CO}_{2}$ is suggested, that is, the peak maximum for the amorphous chain motion ( $\alpha_{a}$ relaxation) shifts to a higher frequency by several orders of magnitude by applying pressurized $\mathrm{CO}_{2}$ up to a few MPa. ${ }^{6,7}$ As the molecular motion of the crystalline chain is not accelerated by applying pressurized $\mathrm{CO}_{2}, \mathrm{CO}_{2}$ is not dissolved inside the crystalline region because of the tight chain packing in the crystalline region. Thus, the plasticization effect of $\mathrm{CO}_{2}$ is not seen in terms of the molecular motion of the crystalline chain, whereas the decline in melting temperature under $\mathrm{CO}_{2}$ is suggested. The decline in melting temperature under $\mathrm{CO}_{2}$ might not be attributed to the accelerated crystalline chain motion inside the crystalline region but to the interaction between $\mathrm{CO}_{2}$ and the PVDF chain near the surface of the crystalline region. Owing to the interaction near the surface, the surface free energy increases and then the melting temperature declines, as demonstrated by Zhang and Handa. ${ }^{4}$

As shown in Figure 3b, the peak for interfacial polarization shifts to a higher frequency and the strength of the peak increases with increasing $\mathrm{CO}_{2}$ pressure. The frequency of the peak maximum obtained from Figure $3 \mathrm{~b}, \omega_{\max }(\mathrm{IP})$, increases with increasing $\mathrm{CO}_{2}$ pressure (Figure 4). Assuming that the crystalline region is spherical with dielectric constant $\varepsilon_{2}$ and conductivity $\sigma_{2}$, and is dispersed in a matrix of the amorphous region with dielectric constant $\varepsilon_{1}$ and conductivity $\sigma_{1}$, the relaxation time of the interfacial polarization, $\tau_{\text {MWS }}$, is given by the Maxwell-Wagner-Sillars equation: ${ }^{17}$

$$
\tau_{\mathrm{MWS}}=\frac{2 \varepsilon_{1}+\varepsilon_{2}}{4 \pi\left(2 \sigma_{1}+\sigma_{2}\right)}
$$

As $\mathrm{CO}_{2}$ molecules are not dissolved in the crystalline region, as demonstrated above, $\varepsilon_{2}$ and $\sigma_{2}$ are unchanged with $\mathrm{CO}_{2}$ pressure. In contrast, $\mathrm{CO}_{2}$ molecules can dissolve in the amorphous region and the chain mobility in that region is accelerated with increasing $\mathrm{CO}_{2}$ pressure. The increase in the upturn with increasing $\mathrm{CO}_{2}$ pressure shown at the low frequency region in Figure 2a suggests an increase in ionic conductivity due to an increase in the mobility of ionic impurities associated with increased chain mobility in the amorphous region. As conductivity increases with increasing chain mobility, $\sigma_{1}$ becomes larger as the amount of dissolved $\mathrm{CO}_{2}$ in the amorphous region increases with increasing $\mathrm{CO}_{2}$ pressure. In contrast, the dielectric constant of PVDF is much higher than that of $\mathrm{CO}_{2}$; that is, the relative permittivity of PVDF is $12^{19}$ and that of $\mathrm{CO}_{2}$ at $100{ }^{\circ} \mathrm{C}$ is 1.19 under pressures below $15 \mathrm{MPa} .{ }^{20}$ Hence, $\varepsilon_{1}$ becomes smaller as the amount of dissolved $\mathrm{CO}_{2}$ in the amorphous region increases with increasing $\mathrm{CO}_{2}$ pressure. Thus, $\sigma_{1}$ increases, whereas $\varepsilon_{1}$ decreases because of the dissolved $\mathrm{CO}_{2}$ in the amorphous region. The increase in $\sigma_{1}$ and the decrease in $\varepsilon_{1}$ yield a decrease in $\tau_{\text {MWS }}$ in Equation (3). The fact that $\omega_{\max }$ (IP) is the inverse of $\tau_{\text {MwS }}$ might explain the increase in $\omega_{\max }$ (IP) with increasing $\mathrm{CO}_{2}$ pressure, as shown in Figures $3 \mathrm{~b}$ and 4.

\section{CONCLUSION}

We found by in situ dielectric relaxation measurement that the shape and frequency of the peak maximum for the $\alpha_{c}$ relaxation of the crystallized PVDF did not change, although pressurized $\mathrm{CO}_{2}$ was applied up to $10 \mathrm{MPa}$. The result suggests that $\mathrm{CO}_{2}$ is not dissolved inside the crystalline region and the crystalline chain motion is not accelerated under $\mathrm{CO}_{2}$. On the other hand, the frequency of the peak maximum for interfacial polarization shifted to a higher frequency with increasing $\mathrm{CO}_{2}$ pressure because of a decrease in the dielectric constant and an increase in the conductivity in the amorphous region with an increase in the amount of dissolved $\mathrm{CO}_{2}$ in the amorphous region.

\section{ACKNOWLEDGEMENTS}

This work was partially supported by the Japan Society for the Promotion of Science (Grant-in-Aid for Scientific Research, No. 20350101).

1 Kazarian, S. G Polymer Processing with Supercritical Fluids. Polym. Sci. Ser. C 42 , 78-101 (2000).

2 Goel, S. K. \& Beckman, E. J Plasticization of poly(methyl methacrylate) (PMMA) networks by supercritical carbon dioxide. Polymer 34, 1410-1417 (1993).

3 Shieh, Y.- T., Su, J.- H., Manivannan, G., Lee, P. H. C., Sawan, S. P. \& Spall, W. D Interaction of supercritical carbon dioxide with polymers. II. Amorphous polymers. J. Appl. Polym. Sci. 59, 707-717 (1996).

4 Zhang, Z. \& Handa, Y. P $\mathrm{CO}_{2}$-assisted melting of semicrystalline polymers. Macromolecules 30, 8505-8507 (1997).

5 Areerat, S., Nagata, T. \& Ohshima, M Measurement and prediction of LDPE/CO solution viscosity. Polym. Eng. Sci. 42, 2234-2245 (2002).

6 Matsumiya, Y., Inoue, T., Iwashige, T. \& Watanabe, H Dielectric relaxation of polymer/ carbon dioxide systems. Macromolecules 42, 4712-4718 (2009).

7 Hirota, S., Tominaga, Y., Asai, S. \& Sumita, M Dielectric relaxation behavior of poly(methyl methacrylate) under high-pressure carbon dioxide. J. Polym. Sci., Part B: Polym. Phys. 43, 2951-2962 (2005).

8 Lian, Z., Epstein, S. A., Blenk, C. W. \& Shine, A. D. Carbon dioxide-induced melting point depression of biodegradable semicrystalline polymers. J. Supercrit. Fluids 39 , 107-117 (2006).

9 Koga, Y. \& Saito, H. Porous structure of crystalline polymers by exclusion effect of carbon dioxide. Polymer 47, 7564-7571 (2006).

10 Oliveira, N. S., Dorgan, J., Coutinho, J. A. P., Ferreira, A., Daridon, J. L. \& Marrucho, I. M. Gas solubility of carbon dioxide in poly(lactic acid) at high pressures. J. Polym. Sci., Part B: Polym. Phys. 44, 1010-1019 (2006).

11 Shenoy, S. L., Fujiwara, T. \& Wynne, K. J. Quantifying plasticization and melting behavior of poly(vinylidene fluoride) in supercritical $\mathrm{CO}_{2}$ utilizing a linear variable differential transformer. Macromolecules 36, 3380-3385 (2003).

12 Nakagawa, K. \& Ishida, Y. Annealing effects in poly(vinylidene fluoride) as revealed by specific volume measurements, differential scanning calorimetry, and electron microscopy. J. Polym. Sci., Polym. Phys. 11, 2153-2171 (1973).

13 Saito, H. \& Stühn, B. Exclusion of noncrystalline polymer from the interlamellar region in poly(vinylidene fluoride)/poly(methyl methacrylate) blends. Polymer 35, 475-479 (1994).

14 Hoffman, J. D., Williams, G. \& Passaglia, E Analysis of the $\alpha, \beta$, and $\gamma$ relaxations in polychlorotrifluoroethylene and polyethylene: dielectric and mechanical properties. J. Polym. Sci., Part C 14, 173-235 (1966). 
15 Boyd, R. H. Relaxation processes in crystalline polymers: experimental behaviour-a review. Polymer 26, 1123-1133 (1985).

16 Kremer, F. \& Schönhals, A. Broadband Dielectric Spectroscopy (Springer-Verlagh, Berlin, 2002).

17 Sillars, R. W. The properties of dielectrics containing semi-conducting particles of various shapes. J. Inst. Electr. Eng. 80, 378-394 (1937).
18 Wurm, A., Soliman, R. \& Schick, C Early stages of polymer crystallization-a dielectric study. Polymer 44, 7467-7476 (2003).

19 Gaul, L. \& Hurlebaus, S. Determination of the impact force on a plate by piezoelectric film sensors. Arch. Appl. Mech. 69, 691-701 (1999).

20 Keyes, F. G. \& Kirkwood, J. G The dielectric constant of carbon dioxide as a function of temperature and density. Phys. Rev. 36, 754-761 (1930). 